\title{
Archipel
}

ARCHIPEL Études interdisciplinaires sur le monde insulindien

$98 \mid 2019$

Varia

\section{Bernard Banoun, Isabelle Poulin et Yves Chevrel (dir.), Histoire des traductions en langue française}

Jean-Claude Trutt

\section{(2) OpenEdition}

1 Journals

Édition électronique

URL : http://journals.openedition.org/archipel/1524

DOI : 10.4000/archipel.1524

ISSN : 2104-3655

Éditeur

Association Archipel

Édition imprimée

Date de publication : 3 décembre 2019

Pagination : 272-275

ISBN : 978-2-910513-82-5

ISSN : 0044-8613

Référence électronique

Jean-Claude Trutt, « Bernard Banoun, Isabelle Poulin et Yves Chevrel (dir.), Histoire des traductions en langue française », Archipel [En ligne], 98| 2019, mis en ligne le 11 décembre 2019, consulté le 18 mars 2021. URL : http://journals.openedition.org/archipel/1524; DOI : https://doi.org/10.4000/archipel. 1524

Association Archipel 
Rocher évoque, de façon succincte mais précise, le contexte javanais de l'époque : contexte géographique, social et historique de Java et de Batavia en cette année 1876 ; composition de l'armée des Indes ; guerres coloniales, en particulier le déroulement de la Guerre d'Aceh jusqu'en 1876, donnant ainsi chair et vraisemblance à l'épisode mystérieux du passage éclair de Rimbaud. L'illustration, excellente, ajoute un sceau de véracité à ce récit, qui se situe à une distance incertaine entre roman et histoire, car, s'il prend parfois la précaution d'indiquer ses hypothèses par des adverbes (" probablement ", " sans doute ")

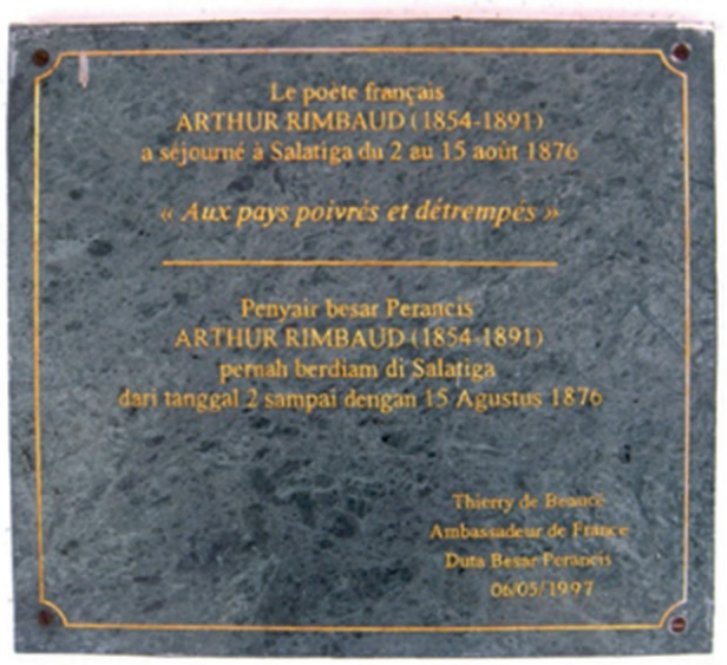

ou des conditionnels, l'auteur en présente d'autres comme des faits avérés.

Un épilogue rapporte la pose, en 1997, par l'ambassadeur de France de l'époque, d'une plaque commémorative sur un bâtiment d'époque coloniale de Salatiga. Épisode piquant, car le «collaborateur» anonyme de l'ambas-sadeur n'est pas anony-me pour tout le monde, et aussi parce que commémorer solennellement, avec les honneurs de la République, un Français engagé dans une armée coloniale étrangère et déserteur de surcroit, ne manque pas d'ironie.

Le livre de Jamie James a connu un certain succès ; la traduction française, en 2012, a trouvé des échos dans la presse. Le petit ouvrage de Rocher, par contre, qui a les dimensions d'un article plutôt que d'un livre et qui est commercialisé en impression à la demande, risque de passer inaperçu, alors qu'il est, en ce qui concerne l'épisode javanais, plus objectif et plus solide. Il mériterait de figurer en bonne place dans l'océan des études rimbaldiennes.

Henri Chambert-Loir

Bernard Banoun, Isabelle Poulin et Yves Chevrel (dir.), Histoire des traductions en langue française, 4ème vol., XX siècle. Paris : Verdier, 2019, 1.920 p., ISBN : 978-2-86432-019-5.

C'est en mai cette année qu'est paru le $4^{\text {e }}$ volume d'une entreprise de longue haleine, qui a débuté en 2012 et que Nicolas Weill, dans un article du 
Monde des Livres daté du 28 juin 2019, qualifie de «monumentale $»^{10}$. Et, effectivement, ce dernier ouvrage est impressionnant : plus de 1900 pages, 4300 traducteurs cités dans l'index et un très grand nombre de thèmes abordés, souvent tout-à-fait originaux, comme la traduction de $\mathrm{BD}$, de livrets d'opéra, de dialogues de cinéma et, bien sûr, de tout ce qui est sciences humaines. Beaucoup de réflexions sur la technique même de la traduction, la traduction poétique par exemple, et l'évolution de la traductologie à partir des années 60. Cela est peut-être l'un des thèmes les plus intéressants (on y explicite, par exemple, les idées de Georges Mounin, l'inventeur des Belles Infidèles ${ }^{11}$, d'Antoine Berman et d'Henri Meschonnic), d'autant plus que cette nouvelle façon de réfléchir à l'art de la traduction est caractéristique du $\mathrm{XX}^{\mathrm{e}}$ siècle. Comme est aussi propre à ce siècle, disent Bernard Banoun et Isabelle Poulin dans leur postface intitulée Bilan, la « formidable extension dans le temps et dans l'espace des ouvrages - et des domaines - traduits ».

Pourtant, si l'on cherche la trace de la Malaisie et de l'Indonésie dans cette Histoire, on reste malheureusement sur sa faim. On a beau chercher, deux noms en tout et pour tout parmi les traducteurs : Georges Voisset pour ses Sonorités pour adoucir les soucis ${ }^{12}$, ouvrage cité comme exemple d' " échantillon unique de littérature malaise », dans la collection Connaissance de l'Orient, et Elisabeth D. Inandiak, citée à propos de « l'unique version française d'une œuvre en javanais, l'immense poème du Livre de Centhini » ${ }_{13}^{13}$ qu'elle a « adapté » (p. 590). FrançoisRené Daillie, qui a, comme Georges Voisset, accompli un travail considérable pour faire connaitre le pantoun véritable, le quatrain court, après qu'Henri Fauconnier, dans son chef d'œuvre Malaisie de 1930, en eût démontré la beauté et l'originalité, est cité uniquement pour une traduction de la poétesse autrichienne Ingeborg Bachmann (p. 638)! On ne trouve nulle part les noms de Louis-Charles Damais, Denys Lombard, Laurent Metzger, Monique Zaini-Lajoubert et de tant d'autres recensés dans l'essai sur la littérature indonésienne en traduction française ${ }^{14}$ qu'Henri Chambert-Loir a publié dans le Bulletin de l'École française d'Extrême-Orient. Et bien sûr : aucun auteur malais ou indonésien.

10. « $\mathrm{Au} \mathrm{XX} \mathrm{X}^{\mathrm{e}}$ siècle, le traducteur sort de l'ombre. Le quatrième tome de la monumentale Histoire des traductions en langue française montre la reconnaissance progressive du traducteur comme auteur au cours du siècle dernier », Nicolas Weill, Le Monde, vendredi 28 juin 2019.

11. Georges Mounin, Les Belles infidèles - Essai sur la traduction, Cahiers du Sud, 1955.

12. Sonorités pour adoucir le souci. Poésie traditionnelle de l'archipel malais, traduit du malais, présenté et annoté par Georges Voisset, Gallimard, Connaissance de l'Orient, 1996.

13. Elizabeth D. Inandiak, Les Chants de l'île à dormir debout. Le Livre de Centhini, Gordes : Les Editions du Relié, 2002.

14. «La littérature indonésienne en traduction française », BEFEO, 104 (2018), p. 205-239. 
Donc, nulle mention du plus grand des écrivains indonésiens, Pramoedya Ananta Toer, dont les premiers romans et nouvelles ${ }^{15}$ ont été écrits dans les années 50 et comptent parmi les premiers à avoir été publiés après l'adoption officielle de la langue malaise comme langue nationale de l'Indonésie.

Et nulle mention du pantoun malais. Qui l'aurait pourtant mérité. Alors que le hasard a voulu que Victor Hugo découvre une variante, le pantoun lié ou pantun berkait, et mette à la mode un type de poème semblable baptisé, à la suite d'une erreur typographique, pantoum, voilà que François-René Daillie et Georges Voisset remettent le pantoun original à l'honneur et en traduisent entre 1985 et 2000 peut-être un millier (rien que La Lune et les étoiles ${ }^{16}$ de Daillie en contient déjà 500). On trouvera sur le site pantun-sayang-afp. fr ( «le site des amis francophones du pantoun ») une large bibliographie réalisée par Georges Voisset et intitulée bibliopantoun, répertoriant systématiquement, non seulement les traductions de pantouns, mais toutes les « références relatives au pantoun en tant que genre poétique universel autonome ». Les lecteurs de l'Histoire des traductions en langue française au XX' siècle n'en sauront rien. Alors qu'ils entendront au moins parler du hain teny malgache (p. 261). Il est vrai que leur traducteur et interprète était Jean Paulhan...

Bien sûr, ces considérations n'enlèvent rien aux mérites de cette œuvre considérable, à laquelle ont collaboré près de 200 chercheurs et qui est passionnante à parcourir. Elles ont pour seul but de rappeler l'étrange silence fait en France sur les cultures de deux pays dont l'un est le quatrième plus peuplé au monde. L'une des raisons de ce désintérêt est peut-être le fait que nous sommes restés culturellement attachés à notre ancien empire colonial et que l'Asie du Sud-Est, pour nous, représente toujours l'ancienne Indochine. Il y en a certainement beaucoup d'autres. La responsabilité du monde de l'édition par exemple. Ainsi, le Quatuor de Buru de Pramoedya Ananta Toer a été traduit en anglais dès 1981, en allemand entre 1987 et 2003, en néerlandais dans les années 80 également, alors qu'en France un éditeur a commencé à éditer timidement le premier tome en $2001^{17}$, en le traduisant à partir de l'anglais, puis s'est arrêté, probablement parce que Pram, cette année-là, n'a pas eu le Nobel comme on l'espérait (ce n'est qu'en 2017 qu'un autre éditeur, Zulma, s'est finalement décidé à le traduire en entier à partir de l'original).

15. La Vie n'est pas une foire nocturne, traduction et présentation par Henri ChambertLoir et Denys Lombard, Gallimard, Connaissance de l'Orient, 1993, nouvelles publiées en Indonésie en 1950 et 51 ; Le Fugitif, traduction François-René Daillie, Plon, 1991, original publié en 1950 ; Corruption, traduction Denys Lombard, Arles : Philippe Picquier, 1991, original publié en 1954.

16. François-René Daillie, La Lune et les Etoiles, le Pantoun malais, Paris : Les Belles Lettres, 2000.

17. Le Monde des hommes, traduit à partir de l'anglais par Michèle Albaret-Maatsch, Rivages, Paris, 2001. 
Les petits éditeurs amateurs sont plus courageux, comme c'est souvent le cas en France. L'Association culturelle franco-indonésienne Pasar Malam a publié une douzaine d'ouvrages traduits de l'indonésien en sept ans. Hélas, depuis que sa présidente et fondatrice, Johanna Lederer, a pris sa retraite, l'Association a décidé d'arrêter sa petite maison d'éditions du Banian.

C'est bien dommage.

Jean-Claude Trutt

\section{Philologie}

Jürg Schneider: Vom Gebrauch der Philologie. Der Luzerner Sprachforscher Renward Brandstetter (1860-1942) (On the Use of Philology. The Lucerne Linguist Renward Brandstetter (1860-1942), Harrassowitz Verlag, Wiesbaden, 2019, 163 p., illustrations, ISBN: 9783447112482

This small book is the biography of a Swiss linguist who was an outsider in Indonesian studies. Working as a professor at the local Gymnasium of Lucerne, Brandstetter had a strong interest in philology and used it first to do research in the dialects and folklore of his native Lucerne. On an excursion in 1884 he met the Dutch professor G.K. Niemann, who taught Malay to missionaries and colonial administrators at the Academy in Delft. Brandstetter got interested in Indonesian languages and started to study Malay, Buginese and Makassarese. He was really fascinated by these languages and became a very prolific writer.

Between 1885 and 1937 he published at his own expense many booklets on "Indonesian" languages and literatures between Madagascar and the Philippines. His publications can be divided into several series: In the first series of his Malayo-Polynesian researches, in 1893 to 1898, he did mostly literary translations; from 1902 to 1908 he ventured into comparative studies of Malayo-Polynesian languages; from 1910 to 1915 he published 7 monographs of Indonesian Linguistics. In his third series of publications "We people of the Indonesian Soil" he published 11 monographs; a 12th written in 1940 was published posthumously in 1992 by W. Marschall.

The English linguist Otto Blagden translated four of these papers into English and published them in 1906 in London under the title Introduction to Indonesian Linguistics.

Brandstetter's other publications were only known in the scientific community on the continent. Like Ferdinand Blumentritt (1853-1913), the specialist and advocate of the Philippines from Leitmeritz in Bohemia, who was also a high school professor all his life, Brandstetter didn't have a university position, and therefore was an outsider in the academic world, but he joined many learned societies to engage in a dialogue with scientists in his field. 